\title{
Promising Performance Indicators for Water Desalination and Aqueous Capacitors Obtained by Engineering the Electric Double Layer in Nano- Structured Carbon Electrodes
}

\author{
Tuan A. Ho and Alberto Striolo* \\ University College London \\ Department of Chemical Engineering \\ Torrington Place, London WC1E 7JE, United Kingdom \\ * Email: a.striolo@ucl.ac.uk
}

\begin{abstract}
It is well known that the electric double layer plays important roles in a variety of applications, ranging from biology to materials sciences. Many studied the electric double layer using a variety of techniques, and as a result our understanding is mature, although not complete. Based on detailed understanding, we expect that by manipulating the electric double layer we could advance tremendously applications in the water-energy nexus. This is particularly true for electric double layer capacitors and capacitive desalination devices. However, such manipulation is not straightforward because of a competition of phenomena that occur within the electric double layer itself, including solvation effects, excluded volume phenomena, and ion-ion correlations. Using molecular dynamics simulations, we designed a composite graphene-based electrode to manipulate structural and dynamical properties of the electric double layer. Our design favors the formation of the compact Helmholtz layer. Inherent to our design is that the compact Helmholtz layer not only is atomically thick, but it is also highly mobile in the direction parallel to the charged surface. We suggest here how to exploit the properties of the engineered electric double layer towards developing a new continuous desalination process that combines the advantages of membrane and capacitive desalination processes, reducing their shortcomings. Insights on the molecular mechanisms relevant to the water-energy nexus are provided.
\end{abstract}




\section{Introduction}

Growing worldwide population, changes in weather pattern, and demand for improved standards of living all contribute to shortage of both fresh water and energy (the water-energy nexus). Securing abundant fresh water and energy in economical yet environmentally friendly manners is indeed one of the critical challenges our society faces. ${ }^{1,2}$ A combination of water desalination technologies and renewable energy sources will most likely be needed to win such challenge. Capacitive desalination (CD) promises to purify large amounts of salty water with low energetic consumption. $^{3}$ Energy storage devices, e.g., water-based electric double layer capacitors (EDLCs), are needed to deploy renewables at the large scale. ${ }^{4}$ The performance of both CD and EDLC processes relies on the properties of the electric double layer (EDL) formed near carbonbased electrodes.

Back in 1853, Helmholtz was the first to propose the concept of the compact EDL, ${ }^{5}$ suggesting that the excess surface charge is compensated by courter-ions closely packed near the surface. Although the Helmholtz EDL, with thickness comparable to the radius of solvated ions, ${ }^{6,7}$ was observed in solutions of sufficiently high salt concentrations $(>1 \mathrm{M}),{ }^{8,9}$ the thermal motion of ions ${ }^{9}$ yields the 'diffuse' EDL, as proposed by Gouy ${ }^{10}$ and Chapman, ${ }^{11}$ in which the surface charge is balanced by a cloud of rather mobile counter-ions. The diffuse EDL extends to a thickness, the Debye length, that depends on salt concentration, with lower concentrations leading to larger thicknesses. ${ }^{12}$ In many cases the EDL resembles the 1924 Gouy-Chapman-Stern model, ${ }^{13}$ according to which some ions bind to the charged surface (i.e., the compact layer), and others remain in its vicinity (i.e., the diffuse layer).

The EDL is important in the water-energy nexus because both CD and EDLCs function by accumulating and then releasing ions near charged surfaces and/or pores. In the EDLCs literature the accumulation of ions near the surface is quantified in terms of the electrodes capacitance, $\mathrm{C}$. Borrowing the Gouy-Chapman-Stern model, $\mathrm{C}$ can be estimated as:

$$
\frac{1}{C}=\frac{1}{C_{H}}+\frac{1}{C_{D}} .
$$


In Eq. (1) $C_{H}$ and $C_{D}$ are the capacitance of the Helmholtz layer and that of the diffuse layer, respectively. Note that, based on Eq. (1), the smaller one out of $C_{H}$ or $C_{D}$ dominates C. In general, $\mathrm{C}_{\mathrm{H}}$ is smaller than $C_{D}$, and therefore it is the dominant contribution to $\mathrm{C}^{8,}{ }^{14}$ Increasing $\mathrm{C}_{\mathrm{H}}$, and hence $\mathrm{C}$, improves the EDLCs' energy density. In CD, because it is desired to balance the excess surface charge by counter-ions within a compact layer, augmenting the compact EDL yields the highest charge efficiency. ${ }^{15}$ Under optimal conditions, one charge unit on the electrode removes one ion from a $1-1(\mathrm{NaCl})$ electrolyte solution. ${ }^{16}$ Unfortunately, in carbon-based electrodes the EDL tends to be diffuse, unless narrow pores are used to remove the solvation

shell from the ions. ${ }^{17,18}$ Promoting the formation of the compact EDL in carbon-based electrodes has therefore the potential of positively impacting both EDLC and CD devices.

We introduce here a graphene-based composite electrode that promotes the formation of the atomically thick Helmholtz layer at conditions at which the diffuse layer would be expected. More importantly, in our design the compact layer, not mobile in conventional electrodes, can slip in the direction parallel to the charged surface. Our equilibrium and non-equilibrium molecular dynamics (MD) simulation results suggest promising performance indicators towards advancements in water desalination and energy-storage devices.

\section{Simulation Details}

We considered two electrochemical cells consisting of an electrode pair in contact with an aqueous $\mathrm{NaCl}$ solution of salinity $\alpha \sim 5.45 \mathrm{~g} / 1(0.093 \mathrm{~mol} / \mathrm{l})$ at $300 \mathrm{~K}$. In the first cell ('bare electrode', Fig. 1A), two oppositely charged graphitic layers face across the aqueous solution. This configuration has been studied extensively. ${ }^{19-21}$ In the second cell ('composite electrode', Fig. 1B) the configuration is very similar to that of the bare electrode, except one neutral graphene membrane is placed in front of each charged graphitic electrode, at a distance of 0.7 nm. In each of these graphene membranes we carved two holes, each of diameter $\mathrm{D}=10 \AA$ (Fig. 1C) by removing the necessary carbon atoms from the graphene sheet. The holes allow both ions and water to exchange between the 'side pore', near the graphitic charged surface, and the solution at the center of the cell. We show herein that the graphene membranes effectively modify the structure of the EDL, yielding the unexpected performance in our design. 
As a first approximation, the effect of different functional groups bound to the holes is not quantified here, although it is known to have a strong effect in graphene-based membranes. ${ }^{22,23}$ Experimentally, graphene membranes can be produced by several methods including chemical vapor deposition, ${ }^{24}$ graphene irradiation with a focused electron beam, ${ }^{25}$ and ultraviolet-induced oxidative etching. ${ }^{26}$ The practical arrangement of the graphene membranes near graphitic electrodes requires mechanical aids to prevent the graphene membranes from collapsing on the graphitic electrodes. This collapse would be driven primarily by van der Waals interactions between the graphene layers, and by the tendency of water to evaporate from the region between two hydrophobic surfaces. ${ }^{27}$ Methods similar to the one recently proposed by Huang et al. ${ }^{28}$ could help solve this design issue.
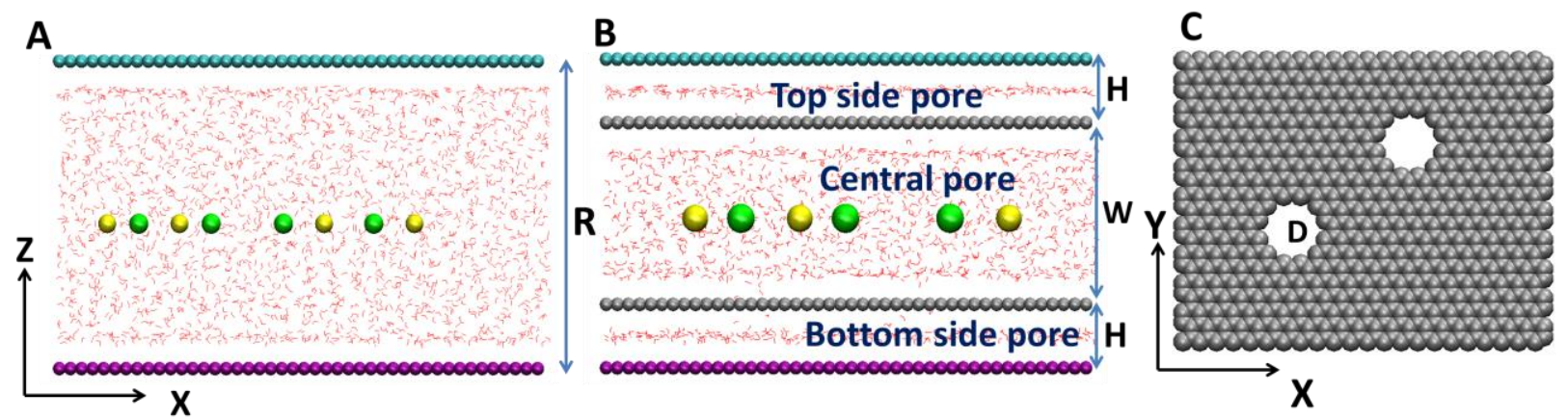

Fig. 1. Schematic representations of the simulated electrochemical cells. In the bare cell (A), the negatively charged (purple) and positively charged (cyan) graphitic electrodes face across the slit pore of width $\mathrm{R}=34 \AA$. In the composite cell $(\mathbf{B})$, four graphene sheets are stacked vertically to create three slitshaped pores: one central pore of width $\mathrm{W}=20 \AA$ and two side-pores (top and bottom), each of width $\mathrm{H}=7 \AA$. The two outermost graphene sheets are charged positively (cyan) and negatively (purple), as in the bare cell, while the two inner graphene sheets (gray) are not charged. These graphene layers contain two holes each, both of diameter $\mathrm{D}=10 \AA(\mathbf{C})$. These graphene sheets act as membranes. In both bare and composite cells the area in the $\mathrm{XY}$ plane is $5.4 \mathrm{x} 4.2 \mathrm{~nm}^{2}$. The simulations are conducted at two surface charge densities $=3.2 \mu \mathrm{C} / \mathrm{cm}^{2}$ and $=4.2 \mu \mathrm{C} / \mathrm{cm}^{2}$, in an effort to replicate realistic experimental conditions. All simulations are initiated by distributing water molecules (shown in red according to the wireframe convention) uniformly throughout the system. The desired number of $\mathrm{Na}^{+}$(yellow) and $\mathrm{Cl}^{-}$ (green) ions are placed within the pore center. The simulated systems are electrically neutral. All simulations are conducted at $300 \mathrm{~K}$. 
To simulate the electrodes, we assign the same partial charge on all carbon atoms (i.e., we impose a constant surface charge density on each electrode). Merlet et al. ${ }^{29,30}$ showed that it is possible to conduct simulations similar to those discussed herein by imposing constant potential across the cell. These authors found that the distribution of electrolytes at the solid-liquid interface is strongly dependent on the algorithm implemented at high surface charge density (i.e., $\left.1.00 \mathrm{e} / \mathrm{nm}^{2}\right)$. At the rather low surface charge densities considered here $\left(0.26 \mathrm{e} / \mathrm{nm}^{2}\right.$ or less $)$, the results are not expected to depend strongly on the algorithm implemented (i.e., constant charge density vs. constant potential).

Because we imposed periodic boundary conditions in all directions, the graphene electrodes are infinitely long along the $\mathrm{X}$ and $\mathrm{Y}$ directions (see Figure 1 for a schematic). Along the $\mathrm{Z}$ direction a large vacuum volume was added above and below the charged graphene layers to minimize undesired interactions between the periodic replicas of our system, which could be caused by long-range electrostatic interactions. ${ }^{31}$ All the graphene surfaces were kept rigid and simulated by the force field proposed by Cheng and Steele. ${ }^{32}$ The simulated bare cells contained 1764 water molecules and 3 ion pairs, while the composite cells contained 2268 water molecules and 4 ion pairs. These compositions yield the salinity $\alpha \sim 5.45 \mathrm{~g} / \mathrm{l}(0.093 \mathrm{~mol} / \mathrm{l})$, which is consistent with that usually employed in capacitive desalination. ${ }^{16}$

Many water models are available in the literature. In a prior publication, we compared the predictions obtained by simulating different water models on graphene. ${ }^{38} \mathrm{We}$ found that the structure of interfacial water predicted implementing SPC/E, TIP4P/2005, SPC/Fw, TIP4P/2005f and SWM4_DP water models is rather similar on neutral, negatively, or positively charged graphene. In this work, water is simulated by implementing the SPC/E model. ${ }^{33} \mathrm{NaCl}$ ions are modeled by the force field proposed by Dang, ${ }^{34}$ without considering polarization effects. Some studies suggest that polarization effects might impact water and ions properties at interfaces. ${ }^{35-37}$ These effects are not investigated in details in this manuscript because, based on our recent analysis, ${ }^{36}$ they should not significantly affect the results presented. In all systems considered here, the temperature is kept constant at $300 \mathrm{~K}$, using the Nose-Hoover algorithm. The equations of motion are integrated using the GROMACS simulation package, version 4.0.7, with the time step of 1 fs. For other simulation details please refer to our previous publications. ${ }^{22,36,39}$ 
Both equilibrium and non-equilibrium simulations were conducted. We conducted our equilibrium all-atom simulations in the NVT ensemble. The simulated systems are equilibrated for $100 \mathrm{~ns}$. Equilibration is considered achieved when the number of ions confined within the side pores remains constant over $50 \mathrm{~ns}$. The results obtained during the last $50 \mathrm{~ns}$ of the equilibrium simulations are used to compute the averages reported herein.

Non-equilibrium simulations were conducted to study the flow of electrolyte solutions inside the electrochemical cells. In these non-equilibrium simulations all water molecules and $\mathrm{NaCl}$ ions are forced to move along the $\mathrm{X}$ direction with constant applied acceleration $2.4 \times 10^{13} \mathrm{~m} / \mathrm{s}^{2}$ (mimicking, e.g., a gravity-driven flow). ${ }^{40-42}$ In these simulations, because the periodic boundary conditions are applied in the direction of flow, the water and ions that exit the system because of the imposed flow enter the pore from the opposite end of the electrode, thus maintaining a constant number of molecules within the simulation cell. Because of the periodic boundary conditions, the simulations do not account for phenomena that could be encountered when water and ions enter or exit the electrodes. The non-equilibrium simulations are conducted until the velocities of water and ions in the $X$ direction remain constant over a period of $100 \mathrm{~ns}$ (i.e., steady states are achieved). Instead of applying acceleration of $2.4 \times 10^{13} \mathrm{~m} / \mathrm{s}^{2}$ to all molecules, an equivalent pressure of $100 \mathrm{MPa}$ can be imposed to drive the flow inside the pores. The large value of the acceleration applied in our simulations, clearly non realistic, is due to computing power limitations. ${ }^{43,44}$ However, because it has been reported that the time scale for fluid flow scales linearly with the applied acceleration, ${ }^{23,45}$ and because fast water transport through graphitic nanopores and carbon nanotubes under small applied pressures was experimentally observed, ${ }^{46,47}$ we expect that small applied pressures can drive the flow through the proposed desalination cell following mechanisms analogous to those discussed below. 

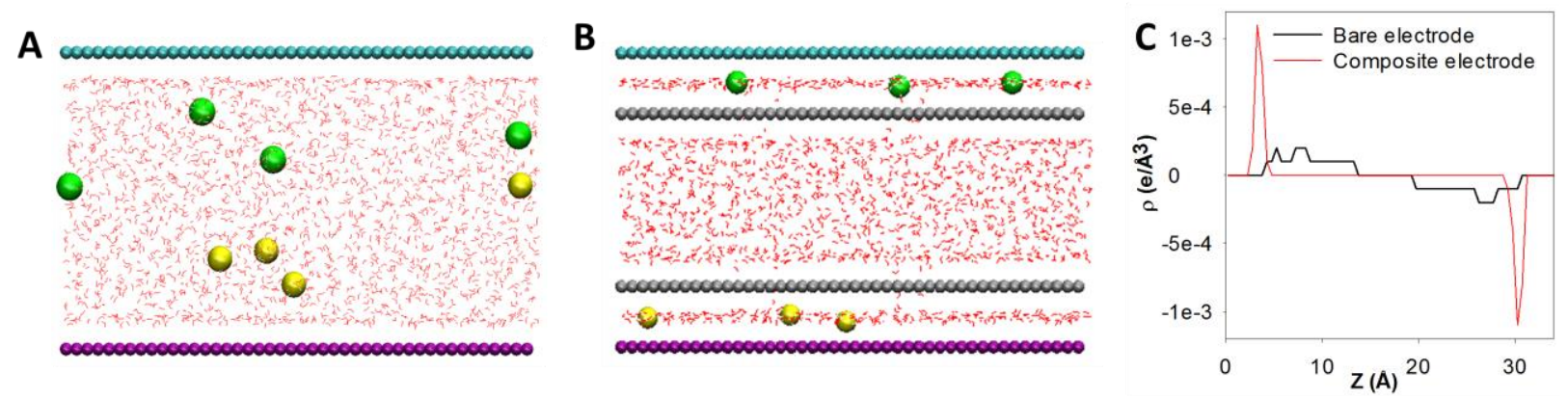

Fig. 2. Representative simulation snapshots at equilibrium for the bare (A) and composite (B) electrochemical cells. These simulations are conducted at the surface charge density $=3.2 \mu \mathrm{C} / \mathrm{cm}^{2}$. The color code is the same as that of Fig. 1. Note that while in the bare cell the ions distribute throughout the entire pore, with counter-ions accumulating near the charged surfaces, in the composite cell the ions are not present within the central pore. Instead they accumulate within the side pores, with $\mathrm{Na}^{+}$ions near the negatively charged surface, and $\mathrm{Cl}^{-}$ions near the positively charged one. Charge density profiles obtained at equilibrium for bare (black) and composite (red) cells (C). The density distributions are consistent with the formation of diffuse EDLs in the bare cell, as expected at the low salinity and low charge density considered in these simulations. In contrast, the high intensity and narrow peaks observed for the composite cell indicate the formation of compact layers centered at $3.5 \AA$ and $3.75 \AA$ near negatively and positively charged electrodes, respectively.

\section{Results and Discussion}

We first investigated how the electrode design affects the EDL in the two electrochemical cells. The surface charge density applied on both electrochemical cells is $=3.2 \mu \mathrm{C} / \mathrm{cm}^{2}$. Visual inspection of the simulation snapshots obtained once equilibration is achieved (Fig. 2A and Fig. 2B) reveals that the graphene membranes strongly affect the distribution of the ions. The counter-ions accumulate near the oppositely charged surface in both electrodes, but yielding diffuse EDLs in the bare cell and compact EDLs in the composite cell. The thickness of EDL can be quantified from the charge density profiles of $\mathrm{NaCl}$ shown in Fig. 2C. Within the bare cell (black line) the thickness (i.e., the distance between the electrode and the end of EDL) of the diffuse EDLs near the negative and positive electrodes are $\sim 13.75 \AA$ and $14.25 \AA$, respectively. In contrast, within the composite cell (red line) the thickness of the compact layers near the negative and positive electrodes are $4.75 \AA$ and $5.25 \AA$, respectively. These data are consistent 
with the formation of compact layers at conditions at which diffuse layers are expected. Because of the presence of the graphene membranes, when the ions migrate from the central pore to the side pores they remain trapped within the side pores instead of returning to the central pore consequently to thermal motion (as observed in the bare cell, see Fig. 3A). The ability of the graphene membranes to keep the ions within the side pores is a crucial feature of our design. If the membrane holes diameter $\mathrm{D}$ is too large, the ions can easily diffuse back from the side pores to the central pore (Fig. 3B). As a side note, we point out that, because of confinement effects, there are 6 and 4 water molecules in the first hydration shell of $\mathrm{Cl}^{-}$and $\mathrm{Na}^{+}$ions, respectively, as opposed to 7 and 6 in bulk aqueous solutions. ${ }^{22,48}$ Clearly, the water molecules in the first hydration shells rearrange in a plane parallel to the pore surfaces (Fig. 3C). Because of this rearrangement, the EDL thickness found in our composite cell is not only much smaller than that of the diffuse layer but also smaller than the thickness of a typical Helmholtz EDL in which the ions remain hydrated. ${ }^{6,7}$
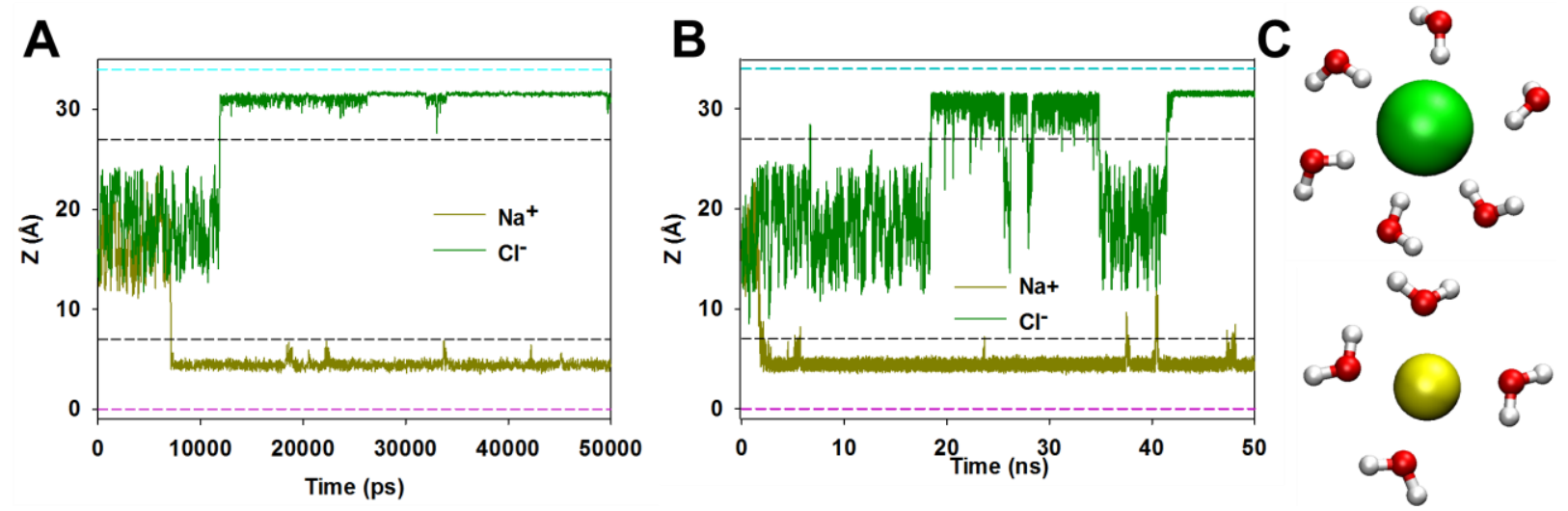

Fig. 3. $\mathrm{Z}$ coordinates of representative $\mathrm{Na}^{+}$and $\mathrm{Cl}^{-}$ions obtained during equilibrium simulations conducted within the composite cell as a function of time when the hole diameter $\mathrm{D}=10 \AA(\mathbf{A})$ and $\mathrm{D}=15 \AA$ (B). The results show that in the case of $\mathrm{D}=10 \AA$ when the ions migrate from the central pore to the side pores (within $\sim 2 \mathrm{~ns}$ in the case of $\mathrm{Na}^{+}$, and $\sim 10 \mathrm{~ns}$ in the case of $\mathrm{Cl}^{-}$), they remain trapped within the side pores for long times. On the contrary, when $\mathrm{D}=15 \AA$ the ions can frequently move between side and central pores. Hydration shells structure of $\mathrm{Cl}^{-}$and $\mathrm{Na}^{+}(\mathbf{C})$ ions within the side-pore of composite cell. 
The latter observation has an important practical consequence as reducing the Helmholtz layer thickness enhances the electrode capacitance $\left[C=\frac{A}{d}, 14,17\right.$ where $\mathrm{A}, \mathrm{d}$, and $\varepsilon$ are surface area, Helmholtz layer thickness, and dielectric constant, respectively]. From the charge density profiles we integrate twice (see Supplemental Information) the Poisson equation 12, 21 $\frac{d^{2}(z)}{d z^{2}}=\frac{(z)}{0}\left[0_{0},(z)\right.$, and $(z)$ are vacuum permittivity, electrical potential, and charge density, respectively] to extract the electrical potential profiles near the charged surfaces (Fig. 4). To conduct this integration we require two appropriate boundary conditions. As first condition, we impose that the electric field in correspondence to the center of the pore is zero $\left(\frac{d \psi}{d z}=0\right.$ at $z=$ $R / 2$, where $\mathrm{R}$ is the pore width). The physical reason underpinning this boundary condition is that at the pore centre the electric field emitted from the negative electrode neutralizes the one emitted from the positive electrode. As the second boundary condition, we impose that the electrical potential is zero at $\mathrm{z}=0$. This condition is arbitrary, and it will not affect the potential difference across the EDL. The electrical potential profiles we obtain allow us to directly calculate the capacitance using the equation $C=-$, where $\sigma$ is the surface charge density and $\psi$ is the potential drop across each EDL (see Table 1). 


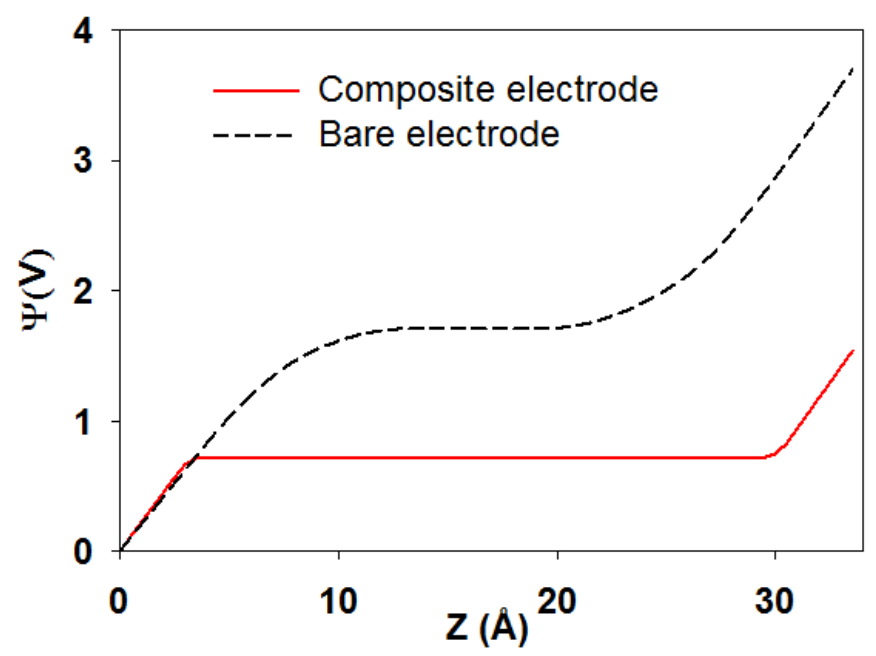

Fig. 4. Electrical potential profiles as a function of distance $\mathrm{Z}$ between two electrodes. The potential drop across the EDL is the difference between the potential found at the interface and that determined at the end of the EDL. These results are obtained by integrating the charge density profiles twice using the Poisson equation following the procedure described in the Supplemental Information.

The capacitance predicted for the composite electrode is much larger than that predicted for the bare electrode, as expected due to the change in the EDL thickness. It is perhaps more important to point out that the capacitance predicted for the composite electrode considered in our simulations is $\sim 70-80 \%$ those reported for electrochemical cells that employ ionic liquids. ${ }^{20,49,50}$ Additional insights regarding the detailed design of the composite cell are presented as Supplemental Information. In short, increasing the system salinity above $5.45 \mathrm{~g} / \mathrm{l}$ does not affect the number of ions in the side pores (Fig. S1), and hence the capacitance of the Helmholtz EDL. Increasing the side-pore width $\mathrm{H}$ from 7 to $10 \AA$ reduces $\mathrm{C}$ (Fig. S2), but does not compromise the operation of the device. Increasing substantially the diameter D of the holes carved on the neutral graphene membranes can lower the ability of the composite electrode to yield the compact EDL, as discussed in Figure 3. 
Table 1. EDL thickness, potential drop across the EDL (up to the EDL thickness), and capacitance obtained for bare and composite electrodes.

\begin{tabular}{|l|c|c|l|c|c|c|}
\cline { 2 - 7 } \multicolumn{1}{c|}{} & \multicolumn{3}{c|}{ Negatively-charged electrode } & \multicolumn{3}{c|}{ Positively-charged electrode } \\
\cline { 2 - 7 } \multicolumn{1}{c|}{} & $\mathrm{d}_{\mathrm{EDL}}$ & Potential drop & Capacitance & $\mathrm{d}_{\mathrm{EDL}}$ & Potential drop & Capacitance \\
\hline Composite & $4.75 \AA$ & $0.72 \mathrm{~V}$ & $4.44 \mu \mathrm{F} / \mathrm{cm}^{2}$ & $5.25 \AA$ & $0.81 \mathrm{~V}$ & $3.95 \mu \mathrm{F} / \mathrm{cm}^{2}$ \\
\hline Bare & $13.75 \AA$ & $1.71 \mathrm{~V}$ & $1.87 \mu \mathrm{F} / \mathrm{cm}^{2}$ & $14.25 \AA$ & $1.99 \mathrm{~V}$ & $1.6 \mu \mathrm{F} / \mathrm{cm}^{2}$ \\
\hline
\end{tabular}

The compact EDL described in Fig. 2B (composite electrode) is not only atomically thick, but it also slips in the direction of flow parallel to the electrodes (Fig. 5A), as suggested by our nonequilibrium simulations. These simulations are initiated either from the last configuration obtained from the equilibrium simulations (i.e., the ions are inside the side pores, Fig. 2B) or from the configuration where the ions are inside the central pore (Fig. 1B). The results obtained from these different initial configurations do not differ from each other. The non-equilibrium simulations are conducted until the ions relocate inside the side pores and steady-states flow is achieved, as described in the Methods section. The results presented in Fig. 5A indicate that in the side pores the velocity of water molecules is nonzero (thus suggesting hydrodynamic slip), and it undistinguishable from the velocity of the ions (suggesting that the ions move with water). The velocity of $\mathrm{Na}^{+}$ions is larger than that of $\mathrm{Cl}^{-}$ions because of steric effects within the narrow side pores considered in our design. In the center of the device water molecules flow with higher velocity than in the side pores because the pore is wider, as expected. The hydrodynamic slip observed both in the side pores and within the central pore is consistent with prior experimental and modeling observations. ${ }^{45-47,51,52}$ Our results suggest that the slippage of the compact EDLs observed within the composite cell can tremendously improve the operation of $\mathrm{CD}$ devices because $\mathrm{CD}$ is based on the physical adsorption of ions onto charged porous electrodes. In both flow-by $^{3,16}$ and flow-through ${ }^{53}$ processes when salty water is exposed to a pair of fresh electrodes the counter-ions adsorb onto the charged electrodes, and fresh water is produced. However, because once the ions enter the electrodes they remain trapped electrodes, regeneration is necessary, ${ }^{3,16,53}$ and the process is not continuous, unless complex operations are designed (e.g., desalination with wires). ${ }^{54}$ As opposed to existing technologies, the composite cell we 
propose promises the possibility of continuous operation, because there is no need of electrodes regeneration (Fig. 5B). We christened our designed 'continuous electrode-membrane desalination cell'.
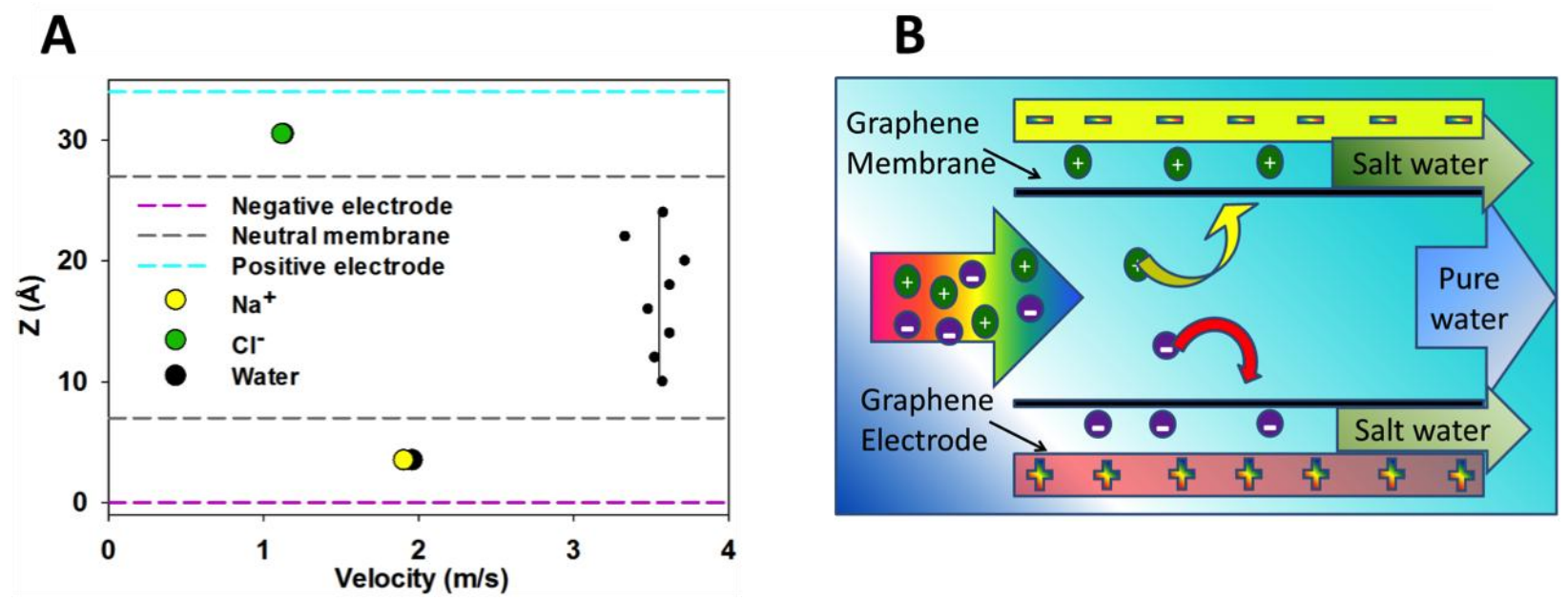

Fig. 5. Velocity of water molecules, $\mathrm{Na}^{+}$and $\mathrm{Cl}^{-}$ions inside the composite electrode cell (A) as obtained from Poiseuille flow simulations. The water flows with the velocity of $\sim 3.5 \mathrm{~m} / \mathrm{s}$ within the central pore. In the bottom side-pore, water and $\mathrm{Na}^{+}$ions flow with the velocity of $1.9 \mathrm{~m} / \mathrm{s}$. In the top side-pore, water and $\mathrm{Cl}^{-}$ions flow with the velocity of $1.2 \mathrm{~m} / \mathrm{s}$. Despite these differences in flow velocities, all the electrolyte solutions slip inside all of the pores in the composite cell, promising continuous CD operation $(\mathbf{B})$.

The continuous electrode-membrane desalination cell we envision is operated as follows: the salty water is fed continuously into the central pore (note that in our non-equilibrium simulations, because of periodic boundary conditions, salty water cannot be fed to the system); during operation the ions diffuse from the central pore to the side pores because of the applied voltages. Compared to existing CD devices, ${ }^{55}$ the envisioned cell has the advantage that the two neutral graphene membranes provide a physical barrier to separate purified from salty water (central and side pores, respectively). Because the compact Helmholtz EDL can slip inside the side pores, and the water in the central pore can flow smoothly through the device, both fresh and salty water can be continually withdrawn from the device. We reiterate that the operation enabled by the proposed design differs substantially compared to flow-through, flow-by, and desalination-with-wires $\mathrm{CD}$ devices in which the ions, once trapped, remain immobilized inside the porous electrodes. ${ }^{16}$ We estimate water permeability $\left(30 \mathrm{~L} / \mathrm{cm}^{2} /\right.$ day/MPa, under the 
assumption that $10 \%$ of the cross surface area of an hypothetical membrane that embeds the proposed cell is constituted by pores) much larger than that obtained from current membranebased water desalination techniques. ${ }^{56}$ At optimum conditions (surface charge density ==+/$4.2 \mu \mathrm{C} / \mathrm{cm}^{2}$ and salinity $\left.<10.9 \mathrm{~g} / \mathrm{l}\right)$ the proposed desalination cell can capture all salty ions within the side pores (100\% rejection), recover $70 \%$ of the salty water initially fed to the system, and yield a charge efficiency of $83 \% .100 \%$ charge efficiency can be obtained at higher salinity using larger pores (Fig. S3). Note that $100 \%$ charge efficiency has been reported in the literature. ${ }^{16}$ Operating the cell will require energy for applying voltages on the electrodes and pumping the salty water through the cell. Because fast water transport through graphitic nanopores and carbon

nanotubes under small applied pressures was experimentally observed, ${ }^{46,47}$ because it has been reported that $\mathrm{CD}$ of brackish water consumes much less energy than reverse osmosis does, $3,57,58$ and because high charge efficiency can be obtained in the engineered cell we propose, we expect the proposed cell to be competitive against existing processes. Unfortunately, quantification of operational costs cannot be conducted reliably at this stage.

\section{Conclusions}

To conclude, we managed to alter the structure of the electric double layer formed within graphitic electrochemical cells. This was achieved by inserting graphene membranes near charged graphitic electrodes. We demonstrated that the ability of manipulating the electric double layer has important implications for the water-energy nexus, as it allows us to both effectively and efficiently store energy and purify water. The compact Helmholtz layer found in the envisioned composite electrochemical cell is not only atomically thick, but it is also able to slip in the direction parallel to the electrodes. The formation of the compact electric double layer significantly enhances the electrodes capacitance, with important implications for the design of electric double layer capacitors. It cannot be overstated that the slippage of the Helmholtz layer is important for the practical realization of the continuous electrode-membrane desalination cells. Based on our simulation results, the proposed desalination cell can be operated continuously, achieving up to $100 \%$ charge efficiency, recovering $70 \%$ of the water initially loaded to the system with $100 \%$ salt rejection at salinity $<10 \mathrm{~g} / \mathrm{l}$, and yielding high water permeability $\left(30 \mathrm{~L} / \mathrm{cm}^{2} /\right.$ day/MPa). 


\section{Acknowledgements}

Generous allocations of computing time were provided by the Oklahoma Supercomputer Center for Education and Research (OSCER) and by the National Energy Resources Supercomputer Center (NERSC). The Authors acknowledge Professor J.M. Prausnitz of UC Berkeley, Professor P.G. Debenedetti of Princeton University, and Dr. Francesco Fornasiero of LLNL for insightful comments.

\section{Supplementary Material}

Additional simulation results concerning the number of $\mathrm{NaCl}$ pairs accumulated within the sidepores as a function of the total number of $\mathrm{NaCl}$ pairs initially present in the salty water (Fig. S1); the effect of side-pore size $\mathrm{H}$ on the capacitance of the composite electrode (Fig. S2 and Table $\mathrm{S} 1$ ); representative simulation snapshot at equilibrium for the composite electrochemical cells in which the side-pore size $H=10 \AA$, salinity $18 \mathrm{~g} / \mathrm{l}$, surface charge density $\mu=+/-4.2 \mu \mathrm{C} / \mathrm{cm}^{2}$ (Fig. S3); and details regarding the integration of the Poisson equation are available free of charge via the Internet at http://pubs.acs.org.

\section{References}

(1) Shannon, M. A.; Bohn, P. W.; Elimelech, M.; Georgiadis, J. G.; Marinas, B. J.; Mayes, A. M. Science and technology for water purification in the coming decades. Nature 2008, 452, 301-310.

(2) Tour, J. M.; Kittrell, C.; Colvin, V. L. Green carbon as a bridge to renewable energy. Nat Mater 2010, 9, 871-874.

(3) Anderson, M. A.; Cudero, A. L.; Palma, J. Capacitive deionization as an electrochemical means of saving energy and delivering clean water. Comparison to present desalination practices: Will it compete? Electrochim Acta 2010, 55, 3845-3856.

(4) Miller, J. R.; Simon, P. Materials science - Electrochemical capacitors for energy management. Science 2008, 321, 651-652.

(5) Helmholtz, H. Ueber einige Gesetze der Vertheilung elektrischer Ströme in körperlichen Leitern mit Anwendung auf die thierisch-elektrischen Versuche. Annalen der Physik 1853, 165, 211-233. 
(6) Bard, A.; Fraulkner, L., Electrochemical Methods: Fundamentals and Applications. John Wiley \& Son: New York, 2001.

(7) Butt, H. J.; Kappl, M., Surface and Interfacial Forces. Wiley-VCH: Weinheim: Germany, 2010.

(8) Huang, J. S.; Qiao, R.; Sumpter, B. G.; Meunier, V. Effect of diffuse layer and pore shapes in mesoporous carbon supercapacitors. J Mater Res 2010, 25, 1469-1475.

(9) Schmickler, W. Electronic effects in the electric double layer. Chem Rev 1996, 96, 3177-3200.

(10) Gouy. On the constitution of the electric charge at the surface of an electrolyte. Cr Hebd Acad Sci 1909, 149, 654-657.

(11) Chapman, D. L. A Contribution to the Theory of Electrocapillarity. Philos Mag 1913, 25, 475-481.

(12) Israelachvili, J., Intermolecular \& Surface Forces. second ed.; Academic Press: New York, 1991.

(13) Stern, O. The theory of the electrolytic double shift. Z Elktrochem Angew P 1924, 30, 508-516.

(14) Wang, H. N.; Pilon, L. Accurate Simulations of Electric Double Layer Capacitance of Ultramicroelectrodes. J Phys Chem C 2011, 115, 16711-16719.

(15) Zhao, R.; Biesheuvel, P. M.; Miedema, H.; Bruning, H.; van der Wal, A. Charge Efficiency: A Functional Tool to Probe the Double-Layer Structure Inside of Porous Electrodes and Application in the Modeling of Capacitive Deionization. J Phys Chem Lett 2010, 1, 205-210.

(16) Porada, S.; Zhao, R.; van der Wal, A.; Presser, V.; Biesheuvel, P. M. Review on the science and technology of water desalination by capacitive deionization. Progress in Materials Science 2013, 58, 1388-1442.

(17) Chmiola, J.; Yushin, G.; Gogotsi, Y.; Portet, C.; Simon, P.; Taberna, P. L. Anomalous increase in carbon capacitance at pore sizes less than 1 nanometer. Science 2006, 313, 1760-1763.

(18) Largeot, C.; Portet, C.; Chmiola, J.; Taberna, P. L.; Gogotsi, Y.; Simon, P. Relation between the ion size and pore size for an electric double-layer capacitor. J Am Chem Soc 2008, 130, 2730-2731.

(19) Fedorov, M. V.; Kornyshev, A. A. Ionic liquid near a charged wall: structure and capacitance of electrical double layer. J Phys Chem B 2008, 112, 11868-72.

(20) Vatamanu, J.; Borodin, O.; Smith, G. D. Molecular Insights into the Potential and Temperature Dependences of the Differential Capacitance of a Room-Temperature lonic Liquid at Graphite Electrodes. J Am Chem Soc 2010, 132, 14825-14833.

(21) Shim, Y.; Kim, H. J.; Jung, Y. Graphene-based supercapacitors in the parallel-plate electrode configuration: Ionic liquids versus organic electrolytes. Faraday Discuss 2012, 154, 249-263.

(22) Konatham, D.; Yu, J.; Ho, T. A.; Striolo, A. Simulation Insights for Graphene-Based Water Desalination Membranes. Langmuir 2013, 29, 11884-11897.

(23) Cohen-Tanugi, D.; Grossman, J. C. Water Desalination across Nanoporous Graphene. Nano Letters 2012, 12, 3602-3608.

(24) O’Hern, S. C.; Boutilier, M. S. H.; Idrobo, J.-C.; Song, Y.; Kong, J.; Laoui, T.; Atieh, M.; Karnik, R. Selective lonic Transport through Tunable Subnanometer Pores in Single-Layer Graphene Membranes. Nano Letters 2014.

(25) Garaj, S.; Liu, S.; Golovchenko, J. A.; Branton, D. Molecule-hugging graphene nanopores. P NatI Acad Sci USA 2013, 110, 12192-12196.

(26) Koenig, S. P.; Wang, L. D.; Pellegrino, J.; Bunch, J. S. Selective molecular sieving through porous graphene. Nat Nanotechnol 2012, 7, 728-732.

(27) Giovambattista, N.; Rossky, P. J.; Debenedetti, P. G. Effect of pressure on the phase behavior and structure of water confined between nanoscale hydrophobic and hydrophilic plates. Phys Rev $E$ 2006, 73.

(28) Huang, H. B.; Song, Z. G.; Wei, N.; Shi, L.; Mao, Y. Y.; Ying, Y. L.; Sun, L. W.; Xu, Z. P.; Peng, X. S. Ultrafast viscous water flow through nanostrand-channelled graphene oxide membranes. Nat Commun 2013, 4. 
(29) Merlet, C.; Rotenberg, B.; Madden, P. A.; Taberna, P. L.; Simon, P.; Gogotsi, Y.; Salanne, M. On the molecular origin of supercapacitance in nanoporous carbon electrodes. Nat Mater 2012, 11, 306310.

(30) Merlet, C.; Pean, C.; Rotenberg, B.; Madden, P. A.; Simon, P.; Salanne, M. Simulating Supercapacitors: Can We Model Electrodes As Constant Charge Surfaces? J Phys Chem Lett 2013, 4, 264268.

(31) Ho, T. A.; Striolo, A. Polarizability effects in molecular dynamics simulations of the graphenewater interface. J Chem Phys 2013, 138, 054117-4 - 054117-9.

(32) Cheng, A.; Steele, W. A. Computer-Simulation of Ammonia on Graphite .1. Low-Temperature Structure of Monolayer and Bilayer Films. J Chem Phys 1990, 92, 3858-3866.

(33) Berendsen, H. J. C.; Grigera, J. R.; Straatsma, T. P. The Missing Term in Effective Pair Potentials. Journal of Physical Chemistry 1987, 91, 6269-6271.

(34) Dang, L. X. Mechanism and Thermodynamics of Ion Selectivity in Aqueous-Solutions of 18Crown-6 Ether - a Molecular-Dynamics Study. J Am Chem Soc 1995, 117, 6954-6960.

(35) Levin, Y. Polarizable lons at Interfaces. Phys Rev Lett 2009, 102, 147803-1 - 147803-4.

(36) Huang, J. Y.; Lo, Y. C.; Niu, J. J.; Kushima, A.; Qian, X. F.; Zhong, L.; Mao, S. X.; Li, J. Nanowire liquid pumps. Nat Nanotechnol 2013, 8, 277-281.

(37) Jungwirth, P.; Tobias, D. J. Ions at the air/water interface. J Phys Chem B 2002, 106, 6361-6373.

(38) Ho, T. A.; Striolo, A. Molecular dynamics simulation of the graphene-water interface: comparing water models. Mol Simulat 2014, 40, 1190-1200.

(39) Ho, T. A.; Striolo, A. Capacitance enhancement via electrode patterning. The Journal of Chemical Physics 2013, 139, 204708-1 - 204708-6.

(40) Kannam, S. K.; Todd, B. D.; Hansen, J. S.; Daivis, P. J. Slip flow in graphene nanochannels. J Chem Phys 2011, 135, 144701-1 - 144701-9.

(41) Thomas, J. A.; McGaughey, A. J. H.; Kuter-Arnebeck, O. Pressure-driven water flow through carbon nanotubes: Insights from molecular dynamics simulation. Int J Therm Sci 2010, 49, 281-289.

(42) Gong, X. J.; Li, J. Y.; Zhang, H.; Wan, R. Z.; Lu, H. J.; Wang, S.; Fang, H. P. Enhancement of Water Permeation across a Nanochannel by the Structure outside the Channel. Phys Rev Lett 2008, 101, 257801-1 - 257801-4.

(43) Ho, T. A.; Papavassiliou, D. V.; Lee, L. L.; Striolo, A. Liquid water can slip on a hydrophilic surface. P Natl Acad Sci USA 2011, 108, 16170-16175.

(44) Lauga E; MP, B.; HA, S., Handbook of Experimental Fluid Dynamics. Springer: New York, 2007.

(45) Holt, J. K.; Park, H. G.; Wang, Y. M.; Stadermann, M.; Artyukhin, A. B.; Grigoropoulos, C. P.; Noy, A.; Bakajin, O. Fast mass transport through sub-2-nanometer carbon nanotubes. Science 2006, 312, 1034-1037.

(46) Holt, J. K.; Hyung Gyu, P.; Yinmin, W.; Stadermann, M.; Artyukhin, A. B.; Grigoropoulos, C. P.; Noy, A.; Bakajin, O. Fast Mass Transport Through Sub-2-Nanometer Carbon Nanotubes. Science 2006, 312, 1034-1037.

(47) Whitby, M.; Quirke, N. Fluid flow in carbon nanotubes and nanopipes. Nat Nanotechnol 2007, 2, 87-94.

(48) Smith, D. E.; Dang, L. X. Computer-Simulations of Nacl Association in Polarizable Water. J Chem Phys 1994, 100, 3757-3766.

(49) Yang, L.; Fishbine, B. H.; Migliori, A.; Pratt, L. R. Molecular Simulation of Electric Double-Layer Capacitors Based on Carbon Nanotube Forests. J Am Chem Soc 2009, 131, 12373-12376.

(50) Shim, Y.; Kim, H. J. Nanoporous Carbon Supercapacitors in an lonic Liquid: A Computer Simulation Study. Acs Nano 2010, 4, 2345-2355.

(51) Kalra, A.; Garde, S.; Hummer, G. Osmotic water transport through carbon nanotube membranes. P Natl Acad Sci USA 2003, 100, 10175-10180. 
(52) Striolo, A. The mechanism of water diffusion in narrow carbon nanotubes. Nano Letters 2006, 6 , 633-639.

(53) Suss, M. E.; Baumann, T. F.; Bourcier, W. L.; Spadaccini, C. M.; Rose, K. A.; Santiago, J. G.; Stadermann, M. Capacitive desalination with flow-through electrodes. Energ Environ Sci 2012, 5, 95119519.

(54) Porada, S.; Sales, B. B.; Hamelers, H. V. M.; Biesheuvel, P. M. Water Desalination with Wires. J Phys Chem Lett 2012, 3, 1613-1618.

(55) Kalluri, R. K.; Konatham, D.; Striolo, A. Aqueous NaCl Solutions within Charged Carbon-Slit Pores: Partition Coefficients and Density Distributions from Molecular Dynamics Simulations. J Phys Chem C 2011, 115, 13786-13795.

(56) Pendergast, M. M.; Hoek, E. M. V. A review of water treatment membrane nanotechnologies. Energ Environ Sci 2011, 4, 1946-1971.

(57) Christen, K. Desalination technology could clean up wastewater from coal-bed methane production. Environmental Science \& Technology 2006, 40, 639-639.

(58) Welgemoed, T. J.; Schutte, C. F. Capacitive Deionization Technology ${ }^{\mathrm{TM}}$ : An alternative desalination solution. Desalination 2005, 183, 327-340. 